\title{
BMJ Open Comparative efficacy and safety of oral anticoagulants for the treatment of venous thromboembolism in the patients with different renal functions: a systematic review, pairwise and network meta-analysis
}

Xiaole Su, ${ }^{1,2}$ Bingjuan Yan, ${ }^{2}$ Lihua Wang, ${ }^{2}$ Hong Cheng, ${ }^{1}$ Yipu Chen (1) ${ }^{1}$

To cite: Su X, Yan B, Wang L, et al. Comparative efficacy and safety of oral anticoagulants for the treatment of venous thromboembolism in the patients with different renal functions: a systematic review, pairwise and network meta-analysis. BMJ Open 2022;12:e048619. doi:10.1136/ bmjopen-2021-048619

- Prepublication history and additional supplemental material for this paper are available online. To view these files, please visit the journal online (http://dx.doi.org/10.1136/ bmjopen-2021-048619).

Received 02 January 2021 Accepted 24 January 2022

Check for updates

(c) Author(s) (or their employer(s)) 2022. Re-use permitted under CC BY-NC. No commercial re-use. See rights and permissions. Published by BMJ.

'Department of Nephrology, Beijing Anzhen Hospital, Capital Medical University, Chaoyang District, Beijing, China ${ }^{2}$ Department of Nephrology, Shanxi Medical University Second Hospital, Shanxi Kidney Disease Institute, Taiyuan, Shanxi, China

Correspondence to

Dr Yipu Chen;

chen_yipu@163.com

\section{ABSTRACT}

Objectives To compare the efficacy and safety of direct oral anticoagulants (DOACs) in patients with venous thromboembolism (VTE) and different renal functions. Design Systematic review containing pairwise and Bayesian network meta-analysis of randomised controlled trials (RCTs).

Data sources MEDLINE, EMBASE and Cochrane Library. Eligibility criteria RCTs reporting the efficacy and safety outcomes of DOACs in different creatinine clearance ( $\mathrm{CrCl})$ subgroups.

Data extraction and synthesis Data extraction and quality assessment were undertaken by two independent reviewers. Data were pooled using the DerSimonian-Laird method in pairwise meta-analysis. Network meta-analysis within a Bayesian framework was conducted.

Results Data from 10 RCTs were included. In the treatment of acute VTE, DOACs did not significantly reduce recurrent VTE or VTE-related death $(\mathrm{OR}, 0.96 ; 95 \% \mathrm{Cl}, 0.82$ to 1.11 ) but significantly reduced bleeding events $(0.76$, 0.68 to 0.90 ) compared with warfarin. In the extended treatment of VTE, DOACs produced significant benefits in recurrent VTE or VTE-related death $(0.23,0.16$ to 0.29$)$, but significantly increased bleeding events $(1.86,1.04$ to 3.33$)$ compared with placebo/aspirin. There were no significant differences in efficacy and safety of DOACs among the three $\mathrm{CrCl}$ stratified subgroups in acute and extended treatment of VTE ( $p$ for subgroup heterogeneity $>0.1$ ). Bayesian network meta-analysis suggested that apixaban $2.5 \mathrm{mg}$ and $5 \mathrm{mg}$ two times per day were associated with a lower risk of bleeding than dabigatran, rivaroxaban, warfarin and aspirin in the subgroup with $\mathrm{CrCl}>80 \mathrm{~mL} /$ $\min$.

Conclusions For the treatment of acute VTE, DOACs are similar to warfarin in reducing recurrent VTE and VTErelated death but are significantly superior to warfarin in reducing the risk of bleeding. For the efficacy and safety of DOACs across different $\mathrm{CrCl}$ stratifications (30-50, 50-80 and more than $80 \mathrm{~mL} / \mathrm{min}$ ), no significant difference was found. In light of minimal evidence, apixaban might be associated with a lower risk of bleeding in patients with VTE and $\mathrm{CrCl}>80 \mathrm{~mL} / \mathrm{min}$.
Strengths and limitations of this study

- The systematic review, pairwise and network meta-analysis included 10 high-quality randomised controlled trials comprising 37298 patients and attempted to assess the efficacy and safety of direct oral anticoagulants (DOACs) in the patients with venous thromboembolism (VTE) and different renal functions.

- Data were classified and pooled based on the creatinine clearance $(\mathrm{CrCl})$ levels in patients receiving acute or extended treatment of VTE.

- Network meta-analysis within a Bayesian framework was conducted to explore the relative efficacy and safety profiles of different DOAC interventions in three $\mathrm{CrCl}$ stratifications and to attempt to explain partly the source of heterogeneity in pairwise meta-analysis.

- The Grading of Recommendations Assessment, Development and Evaluation guidelines and the Confidence in Network Meta-analysis internet application were used to determine the strength of evidence in pairwise and network meta-analysis.

- The inadequate sample size and lower event rate in patients with mild to moderate renal impairment (CrCl 30-50 and 50-80 mL/min, respectively) might affect the results of our research.

\section{PROSPERO registration number CRD42018090896.}

\section{INTRODUCTION}

With a prevalence reaching $10.5 \%-13.1 \%$, the incidence of chronic kidney disease (CKD) is increasing. ${ }^{1}$ Venous thromboembolism (VTE), with an estimated incidence of 0.7-1.4 per 1000 person-years, ${ }^{2}$ which encompasses deep vein thrombosis (DVT) and pulmonary embolism (PE), is a common and potentially fatal disease. There is an increased risk of VTE in patients with nephrotic syndrome, ${ }^{3}$ 
those receiving maintenance dialysis ${ }^{4}$ and kidney transplant recipients. ${ }^{5}{ }^{6}$ In a large prospective cohort study, a glomerular filtration rate (GFR) of less than $45 \mathrm{~mL}$ / min was associated with a 2.13-fold increased risk of VTE compared with a GFR of more than $90 \mathrm{~mL} / \mathrm{min}^{7}$

The introduction of direct oral anticoagulants (DOACs), including direct inhibitors of thrombin (dabigatran) or factor Xa (rivaroxaban, apixaban, edoxaban), has changed the landscape of VTE treatment. Based on several landmark randomised controlled trials (RCTs), ${ }^{8-11}$ the 2014 European Society of Cardiology Guidelines on acute PE suggested that rivaroxaban, apixaban or dabigatran should be considered as an alternative to vitamin $\mathrm{K}$ antagonist (VKA) during extended oral anticoagulation (OAC) therapy. ${ }^{12}$ The 2016 American College of Chest Physicians Treatment Guideline for VTE suggested DOACs (dabigatran, rivaroxaban, apixaban or edoxaban) over VKA therapy in patients with VTE and no cancer. ${ }^{13}$

The different pharmacokinetic characteristics of the four DOACs, including the half-life, the elimination process, the administration and the fluctuation of plasma concentrations, might result in different efficacy and safety profiles, especially for the patients with VTE and impaired renal function. In patients with different renal functions, there are currently no RCTs to directly compare the efficacy and safety of the different DOAC regimens, leading to uncertainty in the selection of clinical treatment regimens. Therefore, whether there is a relatively optimal DOAC treatment regimen in patients with VTE and impaired renal function is a prominent issue.

In this systematic review, our aim was to synthesise all the available data from RCTs and then evaluate the therapeutic benefits and adverse effects of DOACs in patients with VTE stratified by different creatinine clearance $(\mathrm{CrCl})$ levels. Furthermore, we attempted to explore whether there is heterogeneity among DOACs by means of a network meta-analysis within a Bayesian framework.

\section{METHODS}

\section{Data sources and searches}

This systematic review is performed according to a prespecified protocol ${ }^{14}$ registered at the International Prospective Register of Systematic Reviews (CRD42018090896), and the report is in line with Preferred Reporting Items for Systematic Reviews and Meta-Analyses guidelines. ${ }^{15}$ We searched MEDLINE via Ovid, EMBASE via Ovid and the Cochrane Library database (before July 2019) for RCTs (see online supplemental item 1 for full search terms). The ClinicalTrials.gov website was also searched for RCTs that were registered as completed but not yet published. If a trial was published in more than one publication, we used the most detailed publication.

\section{Study selection and outcome definition}

We included RCTs of adult patients with VTE (DVT, PE or both) treated with DOACs (dabigatran, rivaroxaban, apixaban or edoxaban), which reported outcomes in different renal function subgroups. The acute and extended treatment of VTE were both included in our analysis. The control groups included anticoagulantcontrol group (using warfarin as a control, including warfarin alone and enoxaparin followed by warfarin) and non-anticoagulant-control group (using aspirin or placebo as a control). All trials must have an assessment of the efficacy and safety outcomes of DOACs. The efficacy outcome included recurrent VTE and VTE-related death. The safety outcome included major bleeding and clinically relevant non-major bleeding, which were defined individually by each trial. The definitions of efficacy outcome and safety outcome in every trial are presented in online supplemental table 1 . The $\mathrm{CrCl}$ was calculated by Cockcroft-Gault formula in all trials, which were expressed as $\mathrm{mL} / \mathrm{min}$.

\section{Data extraction and quality assessment}

Published reports were obtained for each eligible trial, and relevant information was extracted into a spreadsheet by investigator pairs on the basis of methodological and clinical experience. We used the new Cochrane risk of bias tool for RCTs to assess methodological quality of each study. ${ }^{16}$ The literature search, study selection, data extraction and quality assessment were undertaken independently by two authors (XS and BY) using a standardised approach according to the predefined protocol. Disagreement was resolved by consensus or by a thirdparty arbitrator.

\section{Data synthesis and analysis}

Data were classified based on the $\mathrm{CrCl}$ levels in patients receiving acute or extended treatment of VTE. The random-effects model was applied to generate the summary values according to DerSimonian-Laird method ${ }^{17}$ and the CIs according to Knapp-Hartung modified method. ${ }^{18}$ All of the above operations were run using the software Stata V.12.0 (StataCorp). ORs and 95\% CIs of individual study were calculated from event numbers and the total population at risk extracted from each trial. The percentage of variability across studies attributable to heterogeneity beyond chance was estimated using the $\mathrm{I}^{2}$ statistic. $X^{2}$ test was used to assess the between-subgroup heterogeneity.

Four DOACs were pooled as a whole and compared with controls in pairwise meta-analysis. Network metaanalysis within a Bayesian framework was conducted to explore the relative efficacy and safety profiles of different OAC interventions and to attempt to explain partly the source of heterogeneity in pairwise meta-analysis. Relative effects of different OACs were measured by OR and its $95 \%$ credible intervals. The above operations are run by WinBUGS V.1.4.3 and the R2WinBUGS package of the $\mathrm{R}$ software V.3.1.1. We used non-informative priors with vague normal (mean, 0; variance, 100 000) and uniform (0-5) prior distributions for parameters such as the means and SDs, respectively. ${ }^{19}$ For each analysis, we generated 200000 simulations for each of the two sets of 
different initial values and discarded the first 80000 simulations as the burn-in period. Convergence was reached when Rhat, the potential scale reduction factor, was close to 1 for each of the parameters using the Brooks-GelmanRubin statistic. ${ }^{20}$ We selected the model with a lower value of deviance information criterion (DIC), which suggests a more parsimonious model. ${ }^{21}$ We used the surface under the cumulative ranking curve (SUCRA) probabilities to rank the treatments.

We summarised strength of evidence (SOE) for each outcome individually according to the Grading of Recommendations Assessment, Development and Evaluation guidelines. ${ }^{22}$ The Confidence in Network Meta-analysis internet application was used to determine the confidence in network estimates. ${ }^{23}$ Confidence was initially considered to be high and was maintained or downgraded to moderate, low or very low according to the assessment of the quality of the evidence. ${ }^{24}$

\section{Patient and public involvement}

Patients or the public were not involved in the design, conduct, reporting or dissemination of our research.

\section{RESULTS}

\section{Study search and study characteristics}

A total of 6089 records were identified during our search, and 601 potentially eligible full-text articles were retrieved (figure 1). Overall, 10 RCTs reported in eight articles, ${ }^{8-1025-29}$ comprising 37298 eligible patients, were eventually included in our analysis (see online supplemental table 2 for the details of included studies). All 10 trials were multicentre studies with an average study sample size of 3730 participants. The DOACs in these trials included dabigatran, rivaroxaban, apixaban and edoxaban. In all five trials for the treatment of acute VTE, DOACs were compared with warfarin alone ${ }^{26}$ or enoxaparin followed by warfarin. ${ }^{8-10}{ }^{29}$ For the extended treatment of VTE, DOACs were compared with placebo in three trials, ${ }^{25-27}$ with aspirin in one trial, ${ }^{28}$ and with warfarin in one trial. ${ }^{27}$ To date, no RCT has conducted head-to-head comparisons between different DOACs. Eight RCTs excluded patients with a $\mathrm{CrCl}$ of $<30 \mathrm{~mL} / \mathrm{min}$, whereas the cut-off was slightly lower $(<25 \mathrm{~mL} / \mathrm{min})$ in the two RCTs that included apixaban treatment. ${ }^{85}$ Nine RCTs involved three $\mathrm{CrCl}$ stratifications $(25 / 30-50 \mathrm{~mL} /$ $\min , 50-80 \mathrm{~mL} / \mathrm{min}$ and $>80 \mathrm{~mL} / \mathrm{min}$ ), and one RCT involved two $\mathrm{CrCl}$ stratifications $(30-50$ and $>50 \mathrm{~mL} /$ min).$^{10}$ The detailed results of the risk of bias assessment in the included trials are summarised in online supplemental table 3. The overall methodological quality of the 10 RCTs was moderate to high.

\section{Pairwise meta-analysis}

The efficacy outcome

No statistically significant difference was observed between DOACs as a whole and warfarin for recurrent VTE or VTE-related death in patients with acute VTE (OR, 0.96;

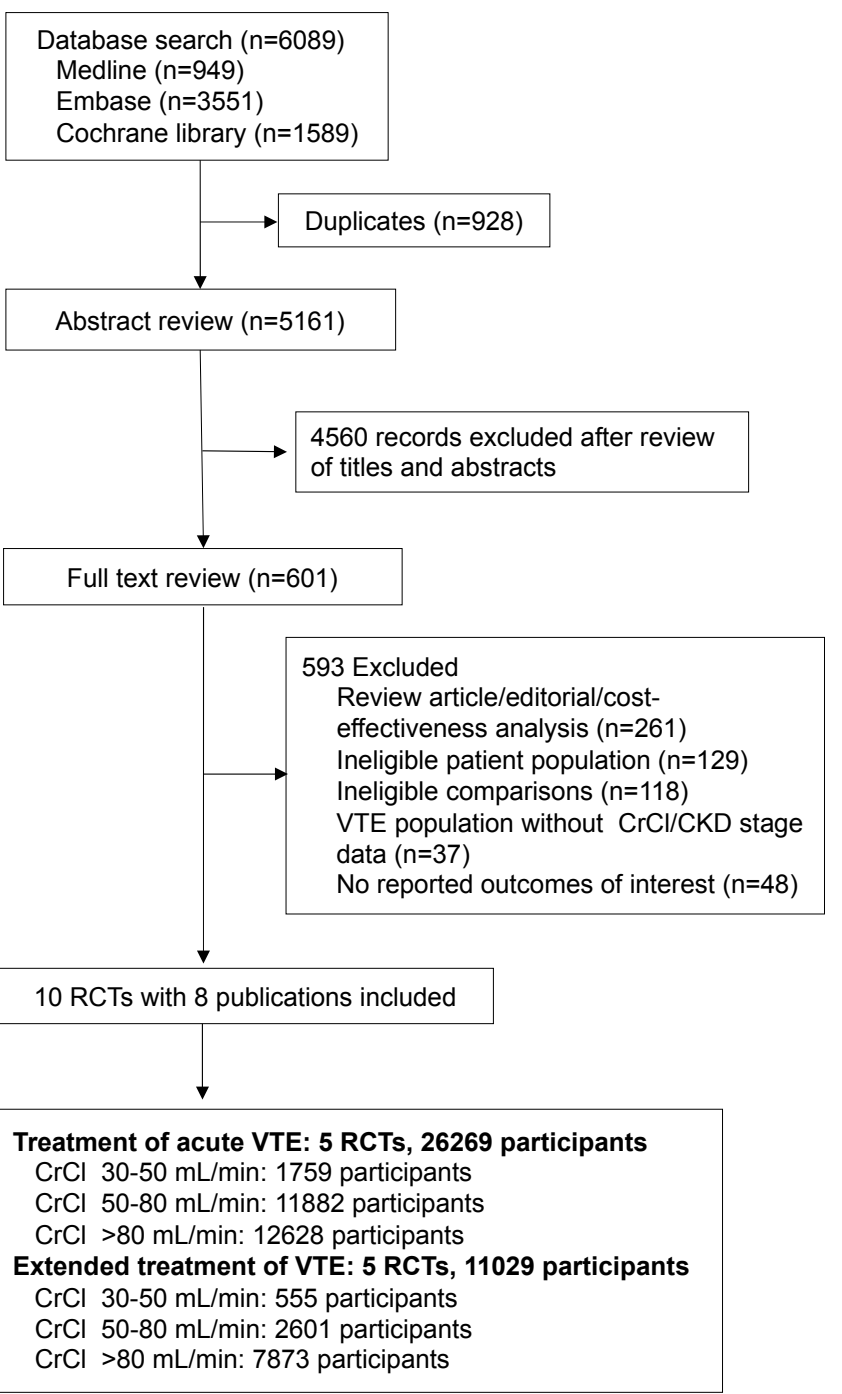

Figure 1 Summary of trial identification and selection. CKD, chronic kidney disease; $\mathrm{CrCl}$, creatinine clearance; $\mathrm{RCTs}$, randomised controlled trials; VTE, venous thromboembolism.

95\% CI, 0.82 to $1.11 ; 5$ RCTs enrolled 26269 patients with 703 events; high SOE) without significant heterogeneity $\left(\mathrm{I}^{2}=0 \%\right)$. In patients with extended treatment for VTE, the use of DOACs produced significant benefits for recurrent VTE or VTE-related death compared with placebo/ aspirin (OR, 0.23 ; 95\% CI, 0.16 to 0.29 ; 4 RCTs enrolled 8205 patients with 260 events; moderate SOE) without significant heterogeneity $\left(\mathrm{I}^{2}=0 \%\right)$.

The effects of DOACs on recurrent VTE or VTE-related death in patients with acute and extended treatment of VTE were not significantly different among the three subgroups of $\mathrm{CrCl}$ stratifications ( $\mathrm{p}$ values for subgroup heterogeneity were 0.45 and 0.78 , respectively). The RE-MEDY trial comparing dabigatran with warfarin is the only trial designed to specifically evaluate the efficacy of DOAC against VKA during the extended treatment of VTE, in which no significant difference was found for recurrent VTE or VTE-related death among the subgroups of different $\mathrm{CrCl}$ stratifications. ${ }^{27}$ 


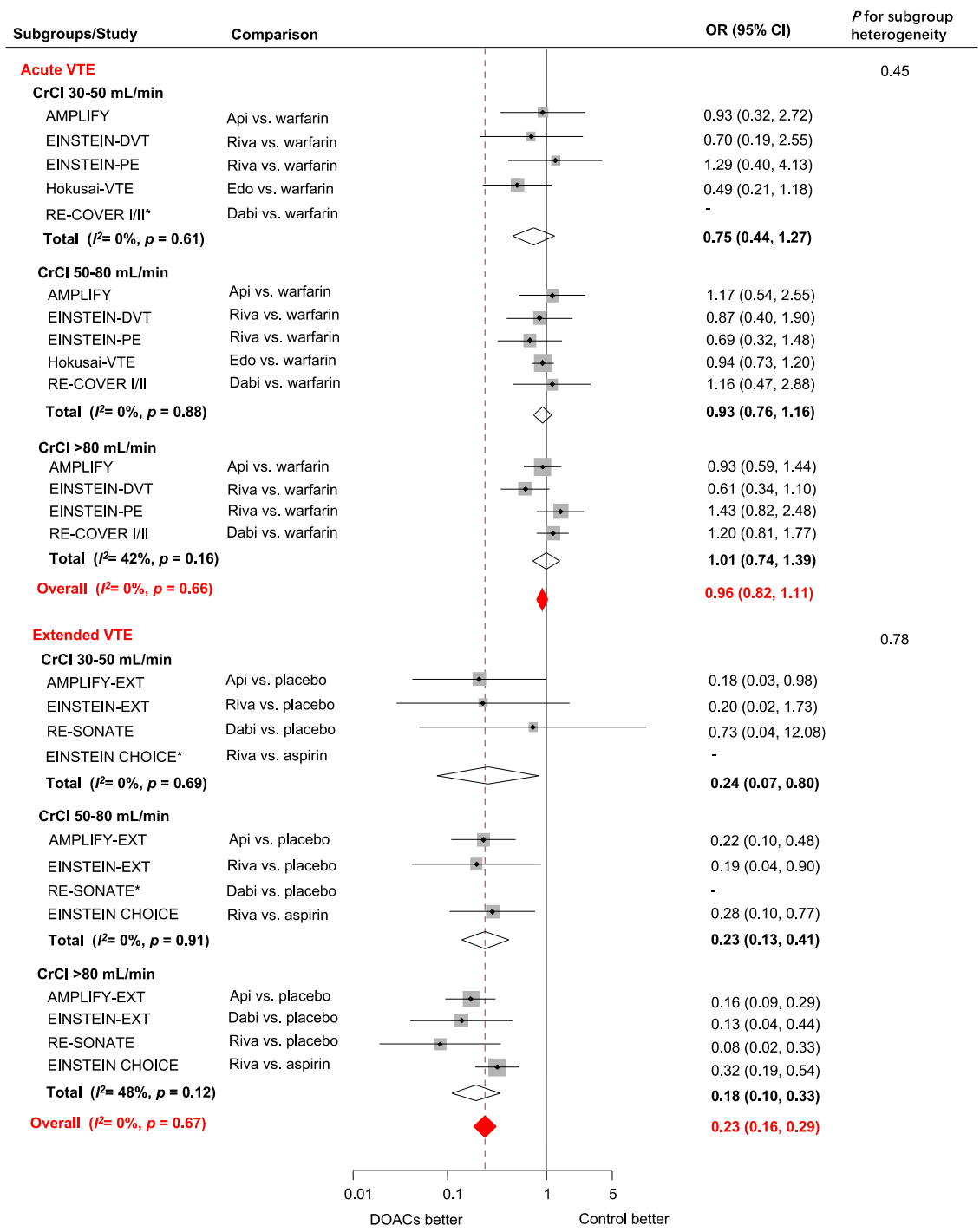

Figure 2 Summary of the efficacy outcome of DOACs therapy according to different CrCl subgroups. *Zero event in at least one treatment arm. Api, apixaban; $\mathrm{CrCl}$, creatinine clearance; Dabi, dabigatran; DOACs, direct oral anticoagulants; Edo, edoxaban; Riva, rivaroxaban; VTE, venous thromboembolism.

The details of the efficacy outcomes are presented in figure 2.

\section{The safety outcome}

In patients with acute VTE, DOACs therapy significantly reduced the risk of bleeding events compared with warfarin (OR, 0.76; 95\% CI, 0.68 to $0.90 ; 26182$ patients with 2473 events; moderate SOE). Conversely, in patients with extended treatment of VTE, the use of DOACs significantly increased the risk of bleeding events compared with aspirin/placebo (OR, 1.86; 95\% CI, 1.04 to $3.33 ; 6859$ patients with 209 events; low SOE). However, significant heterogeneity was found in the safety outcome for both acute and extended VTE treatment $\left(\mathrm{I}^{2}=47.6 \%\right.$ and $55.1 \%$; $\mathrm{p}$ for heterogeneity $=0.02$ and 0.02 , respectively). The subgroup analysis suggested that the main contribution of heterogeneity across studies was from the subgroup of $\mathrm{CrCl}>80 \mathrm{~mL} / \mathrm{min}\left(\mathrm{I}^{2}=85 \%\right.$ in acute treatment and $78.5 \%$ in extended treatment).
In patients with acute and extended treatment of VTE, no significant difference in bleeding events was found among the three subgroups of $\mathrm{CrCl}$ stratifications ( $p$ for subgroup heterogeneity $=0.63$ and 0.21 , respectively).

The details of the safety outcomes are presented in figure 3. The SOE grades (low, moderate or high) and the details of all comparisons and outcomes are summarised and provided in online supplemental table 4 .

\section{Bayesian network meta-analysis}

Network meta-analysis within a Bayesian framework was conducted to explore the relative efficacy and safety of different treatment regimens and to attempt to explain the source of heterogeneity in pairwise meta-analysis. There were seven, nine and eight treatment regimens in patients with VTE with $\mathrm{CrCl}$ of 30-50, 50-80 and more than $80 \mathrm{~mL} / \mathrm{min}$, respectively. The networks of eligible comparisons are shown in online supplemental figure 1. The DIC values from the fixed consistency model were 


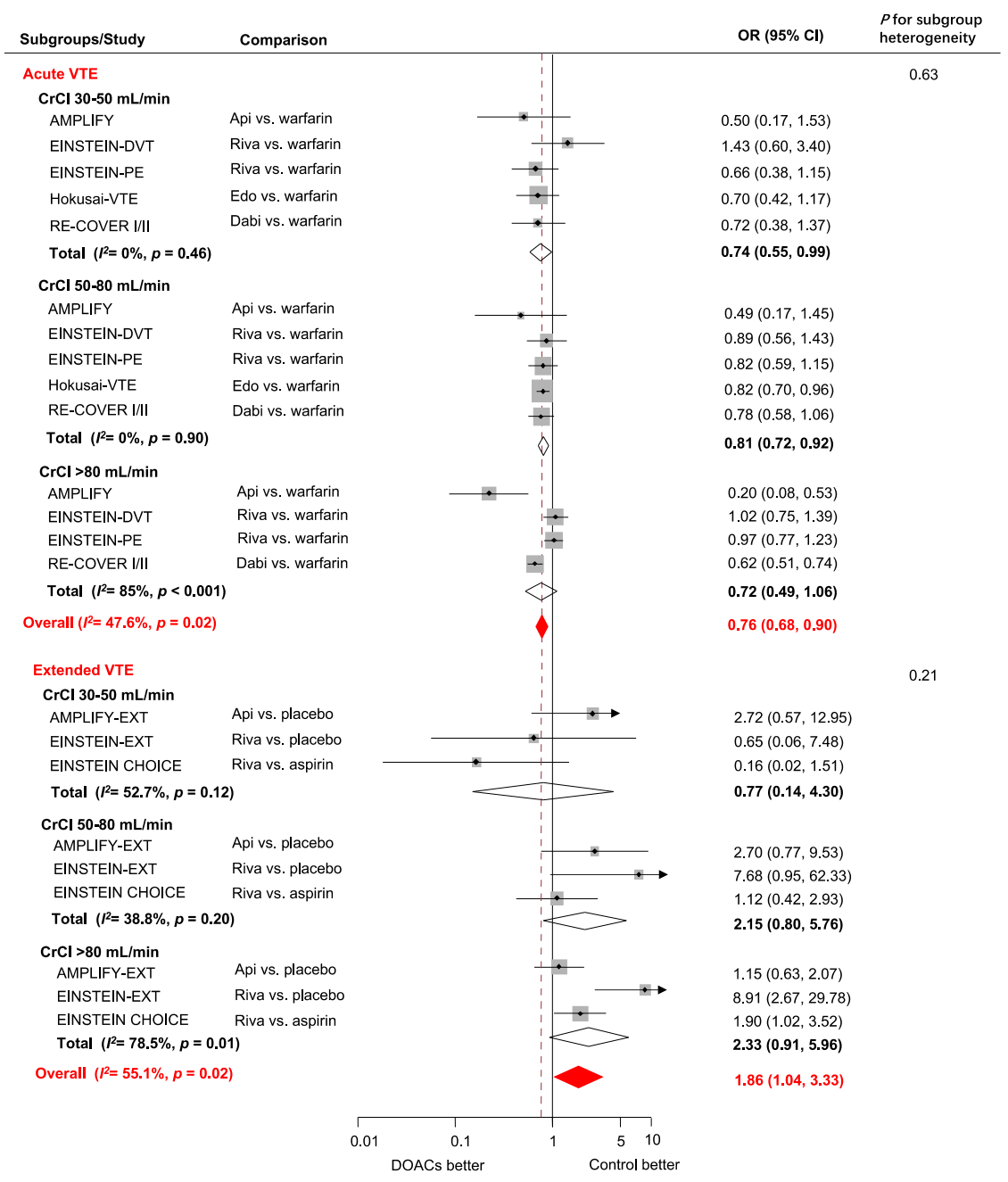

Figure 3 Summary of the safety outcome of DOACs therapy according to different $\mathrm{CrCl}$ subgroups. Api, apixaban; $\mathrm{CrCl}$, creatinine clearance; Dabi, dabigatran; DOACs, direct oral anticoagulants; Edo, edoxaban; Riva, rivaroxaban; VTE, venous thromboembolism.

the lowest, which indicates that it was the preferred model (online supplemental table 5). The primary outcomes of the Bayesian network meta-analysis from the three $\mathrm{CrCl}$ subgroups, including recurrent VTE or VTE-related death and bleeding events, are summarised in figure 4 .

In patients with VTE with $\mathrm{CrCl}$ of $30-50 \mathrm{~mL} / \mathrm{min}$ and $50-80 \mathrm{~mL} / \mathrm{min}$, there was no significant difference in recurrent VTE or VTE-related death and bleeding events between any two OACs (figure 4A,B).

In patients with VTE with $\mathrm{CrCl}$ greater than $80 \mathrm{~mL} /$ min, the significant differences between treatment regimens were mainly from the safety outcomes (figure 4C, the upper triangle with yellow shading). Apixaban $2.5 \mathrm{mg}$ and $5 \mathrm{mg}$ two times per day were associated with reduced bleeding risks compared with the other treatment regimens, including dabigatran $150 \mathrm{mg}$ two times per day, rivaroxaban $10 \mathrm{mg}$ and $20 \mathrm{mg}$ once daily, warfarin and aspirin. No significant difference was found between apixaban $2.5 \mathrm{mg}$ and $5 \mathrm{mg}$ two times per day. Dabigatran $150 \mathrm{mg}$ two times per day was superior to rivaroxaban 20 $\mathrm{mg}$ once daily and warfarin in reducing bleeding events: the ORs (95\% CIs) were 0.61 ( 0.47 to 0.81$)$ and 0.62 (0.51 to 0.74$)$, respectively. These results might partly explain the source of heterogeneity in pairwise meta-analysis, especially when bleeding events were analysed in the subgroup of $\mathrm{CrCl}$ greater than $80 \mathrm{~mL} / \mathrm{min}$.

Because almost all the 95\% CIs of the SUCRAs overlapped widely in all three $\mathrm{CrCl}$ subgroups, the implications of SUCRA might be limited (online supplemental table 6). Only one to two closed loops were formed, and no significant inconsistency was identified (online supplemental figure 2). The confidence ratings for the effect estimates of outcomes are presented in online supplemental table 7 , most of which were low and very low.

\section{DISCUSSION}

How to carry out reasonable anticoagulation therapy for VTE in the patients with renal insufficiency is a very important clinical issue. For acute and extended treatments in patients with VTE with different kidney functions, the subgroup analyses of several large RCTs have 


\begin{tabular}{|c|c|c|c|c|c|c|c|c|}
\hline \multicolumn{9}{|c|}{$\mathrm{A}: \mathrm{CrCl} 30-50 \mathrm{~mL} / \mathrm{min}$} \\
\hline Api $5 \mathrm{mg}$ & $1.20(0.43,3.45)$ & $1.04(0.31,3.45)$ & $1.09(0.33,3.57)$ & $0.66(0.17,2.56)$ & $1.52(0.56,4.55)$ & $0.48(0.11,1.75)$ & - & - \\
\hline $0.97(0.21,2.99)$ & Riva 20 mg & $0.85(0.39 .1 .75)$ & $0.88(0.45,1.75)$ & $0.54(0.09,2.94)$ & $1.27(0.81,1.99)$ & $0.38(0.07,1.85)$ & - & - \\
\hline $5.66(0.38,28.83)$ & $6.79(0.56,26.94)$ & Dabi $150 \mathrm{mg}$ & $1.03(0.47 .2 .27)$ & $0.65(0.09,3.85)$ & $1.47(0.83,2.86)$ & $0.46(0.08,2.63)$ & - & - \\
\hline $2.04(0.39,6.39)$ & $2.55(0.63,7.65)$ & $0.92(0.06,3.73)$ & Edo $30 \mathrm{mg}$ & $0.61(0.11,3.13)$ & $1.41(0.83,2.51)$ & $0.43(0.08,2.22)$ & - & - \\
\hline $0.85(0.03,4.32)$ & $1.23(0.04,6.51)$ & $0.49(0.01,2.61)$ & $0.75(0.02,3.33)$ & Api $2.5 \mathrm{mg}$ & $2.27(0.47,14.29)$ & $0.71(0.15,3.33)$ & - & - \\
\hline $0.85(0.25,2.20)$ & $1.06(0.43,2.35)$ & $0.39(0.03,1.41)$ & $0.52(0.18,1.05)$ & $5.46(0.16,29.1)$ & Warfarin & $0.31(0.06,1.41)$ & - & - \\
\hline $0.19(0.02,0.72)$ & $0.24(0.02,0.85)$ & $0.09(0.01,0.42)$ & $0.13(0.01,0.54)$ & $0.58(0.04,2.09)$ & $0.26(0.02,0.88)$ & Placebo & - & - \\
\hline \multicolumn{9}{|c|}{ B: $\mathrm{CrCl} 50-80 \mathrm{~mL} / \mathrm{min}$} \\
\hline Api $5 \mathrm{mg}$ & $1.71(0.73,6.17)$ & $1.49(0.62,5.56)$ & $1.67(0.71,5.81)$ & $0.98(0.28,6.71)$ & $0.81(0.32,2.48)$ & $1.08(0.34,6.45)$ & $2.04(0.88,7.19)$ & $0.24(0.08,0.91)$ \\
\hline $1.87(0.75,3.89)$ & Riva 20 mg & $0.86(0.59,1.28)$ & $0.96(0.72,1.32)$ & $0.59(0.24,2.14)$ & $0.35(0.11,1.93)$ & $0.64(0.26,2.28)$ & $1.17(0.92,1.55)$ & $0.11(0.03,0.55)$ \\
\hline $1.17(0.39,2.82)$ & $0.68(0.23,1.49)$ & Dabi $150 \mathrm{mg}$ & $1.09(0.77,1.56)$ & $0.66(0.24,2.67)$ & $0.39(0.11,2.09)$ & $0.71(0.26,2.61)$ & $1.34(1.00,1.92)$ & $0.13(0.04,0.69)$ \\
\hline $1.61(0.71,3.32)$ & $0.92(0.47,1.64)$ & $1.56(0.65,3.18)$ & Edo $60 \mathrm{mg}$ & $0.61(0.23,2.39)$ & $0.36(0.11,1.98)$ & $0.65(0.25,2.34)$ & $1.21(1.00,1.53)$ & $0.12(0.04,0.58)$ \\
\hline $1.36(0.09,6.02)$ & $0.72(0.05,2.74)$ & $1.32(0.08,5.89)$ & $0.86(0.06,3.71)$ & Riva 10 mg & $0.42(0.11,4.59)$ & $0.91(0.33,3.17)$ & $1.43(0.54,5.24)$ & $0.13(0.03,1.25)$ \\
\hline $1.03(0.24,2.89)$ & $0.64(0.13,2.02)$ & $0.40(0.03,1.57)$ & $0.72(0.16,2.28)$ & $2.47(0.11,14.52)$ & Api $2.5 \mathrm{mg}$ & $1.03(0.25,9.43)$ & $1.94(0.63,11.36)$ & $0.25(0.07,1.16)$ \\
\hline $0.41(0.04,1.59)$ & $0.22(0.02,0.74)$ & $1.03(0.22,3.47)$ & $0.26(0.03,0.96)$ & $0.41(0.09,1.09)$ & $0.55(0.03,2.52)$ & Aspirin & $1.36(0.54,4.93)$ & $0.13(0.03,1.18)$ \\
\hline $1.49(0.69,2.93)$ & $0.85(0.48,1.38)$ & $1.44(0.63,2.88)$ & $0.94(0.73,1.22)$ & $3.32(0.25,15.36)$ & $2.06(0.43,5.72)$ & $8.87(0.99,37.71)$ & Warfarin & $0.11(0.03,0.49)$ \\
\hline $0.18(0.06,0.38)$ & $0.11(0.03,0.26)$ & $0.18(0.05,0.41)$ & $0.12(0.04,0.29)$ & $0.43(0.02,2.42)$ & $0.22(0.06,0.48)$ & $1.15(0.11,5.36)$ & $0.13(0.04,0.29)$ & Placebo \\
\hline \multicolumn{9}{|c|}{ C: $\mathrm{CrCl}>80 \mathrm{~mL} / \mathrm{min}$} \\
\hline Api $5 \mathrm{mg}$ & $5.26(2.78,14.29)$ & $3.33(1.64,8.33)$ & $3.98(1.82,14.29)$ & $0.65(0.35,1.28)$ & $2.33(1.01,8.33)$ & $5.26(2.71,14.29)$ & $0.68(0.39,1.28)$ & - \\
\hline $1.12(0.32,4.66)$ & Riva $20 \mathrm{mg}$ & $0.61(0.47,0.81)$ & $0.76(0.45,1.39)$ & $0.11(0.04,0.31)$ & $0.44(0.20,1.01)$ & $1.00(0.83,1.20)$ & $0.11(0.05,0.27)$ & - \\
\hline $0.99(0.35,4.49)$ & $0.90(0.29,3.21)$ & Dabi 150 mg & $1.20(0.69,2.44)$ & $0.17(0.07,0.51)$ & $0.71(0.36,1.59)$ & $1.61(1.35,1.96)$ & $0.18(0.09,0.46)$ & - \\
\hline $1.92(0.29,13.21)$ & $1.68(0.40,7.43)$ & $1.91(0.25,11.18)$ & Riva 10 mg & $0.13(0.05,0.43)$ & $0.55(0.29,1.23)$ & $1.22(0.71,2.33)$ & $0.13(0.05,0.41)$ & - \\
\hline $0.98(0.27,3.93)$ & $0.88(0.18,5.14)$ & $0.97(0.17,4.52)$ & $0.52(0.06,4.07)$ & Api $2.5 \mathrm{mg}$ & $3.33(1.33,14.29)$ & $7.69(3.33,24.99)$ & $0.96(0.52,2.08)$ & - \\
\hline $0.44(0.07,2.35)$ & $0.41(0.09,1.38)$ & $0.44(0.05,1.98)$ & $0.38(0.05,1.79)$ & $0.44(0.05,3.08)$ & Aspirin & $2.04(1.05,4.35)$ & $0.22(0.09,0.77)$ & - \\
\hline $1.08(0.39,3.48)$ & $0.96(0.41,2.36)$ & $1.07(0.41,2.34)$ & $0.57(0.12,3.14)$ & $1.09(0.21,5.03)$ & $2.42(0.61,14.97)$ & Warfarin & $0.11(0.05,0.28)$ & - \\
\hline $0.13(0.04,0.41)$ & $0.11(0.03,0.35)$ & $0.13(0.03,0.36)$ & $0.07(0.01,0.36)$ & $0.13(0.03,0.48)$ & $0.29(0.05,1.64)$ & $0.12(0.04,0.34)$ & Placebo & - \\
\hline
\end{tabular}

Figure 4 Summary of the primary results of Bayesian network meta-analysis from the three $\mathrm{CrCl}$ subgroups. (A) $\mathrm{CrCl} 30-50$ $\mathrm{mL} / \mathrm{min}$; (B) $\mathrm{CrCl} 50-80 \mathrm{~mL} / \mathrm{min}$; (C) $\mathrm{CrCl}$ more than $80 \mathrm{~mL} / \mathrm{min}$. Note: column-to-row ORs and $95 \% \mathrm{Cls}$ for incidence of VTE or VTE-related death (on the lower triangle, light blue shading) and bleeding events (on the upper triangle, yellow shading) were shown. An OR $>1$ favours the row-defining treatment and means that the treatment in the row is associated with a lower risk of VTE or VTE-related death and bleeding events than the treatment in the column. To obtain ORs for comparisons in the opposite direction, reciprocals should be taken. Significant results are in bold. Api and dabi were administered two times per day, while the other treatments were administered once daily. Api, apixaban; $\mathrm{CrCl}$, creatinine clearance; Dabi, dabigatran; Edo, edoxaban; Riva, rivaroxaban; VTE, venous thromboembolism.

been performed to explore the effectiveness and safety of DOACs therapy. Our study systematically reviewed these research results, which consisted with $\mathrm{CrCl}$ stratifications. There were two key findings. First, the efficacy and safety of DOACs had no statistically significant change across different $\mathrm{CrCl}$ stratifications (30-50, 50-80 and more than $80 \mathrm{~mL} / \mathrm{min})$. Specifically, in patients with acute VTE, DOACs showed similar efficacy to warfarin for the prevention of recurrent VTE or VTE-related death with fewer bleeding events, while in patients with extended treatment of VTE, DOACs significantly reduced the risk of recurrent VTE or VTE-related death, but a significant increase in bleeding complications was found compared with aspirin/placebo. Second, regardless of acute or extended treatment of VTE, significant heterogeneity for bleeding events was found, especially in patients with a $\mathrm{CrCl}$ of more than $80 \mathrm{~mL} / \mathrm{min}$. The results of the Bayesian network meta-analysis further demonstrated the differences between treatment interventions in patients with a $\mathrm{CrCl}$ of more than $80 \mathrm{~mL} / \mathrm{min}$ : apixaban $2.5 \mathrm{mg}$ or $5 \mathrm{mg}$ two times per day was superior to dabigatran $150 \mathrm{mg}$ two times per day, rivaroxaban $10 \mathrm{mg}$ or $20 \mathrm{mg}$ once daily, aspirin and warfarin, and dabigatran $150 \mathrm{mg}$ two times per day was superior to rivaroxaban $20 \mathrm{mg}$ once daily and warfarin in reducing the risk of bleeding events.

Our systematic review and meta-analysis included only patients with VTE who were treated with OACs, homogenising the research subjects. Furthermore, we attempted to assess the efficacy and safety of DOACs in patients with different renal functions, especially CKD. Subgroup analysis of $\mathrm{CrCl}$ stratification was performed for the first time to explore the influence of the severity of renal insufficiency on the actions of OACs. The source of heterogeneity in the outcome of bleeding events in the pairwise meta-analysis is partly explained in Bayesian network meta-analyses. These findings suggest that in terms of safety, there may be some differences between DOACs. However, there are some limitations that may be 
considered in our systemic review. First, the inadequate sample size and lower event rate in patients with mild to moderate renal impairment ( $\mathrm{CrCl} 30-50$ and $50-80 \mathrm{~mL}$ / min, respectively) might affect the results of our research. Second, our study only included RCT data, which may affect the universality of the results because it is generally believed that the risk of bleeding events in clinical trials is often lower than that in clinical practice, especially in patients with severe renal insufficiency. Third, networks are very sparse, so the majority of SOE grades in network meta-analysis were low or very low. Therefore, the findings of our network meta-analysis study should be viewed as hypothesis generating and need to be confirmed in further studies.

In 2019, Ha et $a l^{30}$ published a systematic review and meta-analysis that compared the benefits and harms of various oral and injection anticoagulants in the treatment of patients with CKD with renal insufficiency $(\mathrm{CrCl}$ of $20-60 \mathrm{~mL} / \mathrm{min}$, estimated GFR $15-60 \mathrm{~mL} / \mathrm{min} / 1.73$ $\mathrm{m}^{2}$ or serum creatinine $\geq 1.5 \mathrm{mg} / \mathrm{dL}$ ). In Ha $e$ t $a l$ s study, the indications for anticoagulation treatment included not only VTE but also other disorders, such as atrial fibrillation, cardiovascular diseases other than atrial fibrillation and thromboprophylaxis in the perioperative period. The results showed that DOACs were similar to VKAs in reducing recurrent VTE or VTE-related death in the treatment of acute VTE. In terms of reducing the risk of major bleeding, an analysis combining all indications (not just VTE) showed that DOACs were superior to VKAs, but the difference had not yet reached statistical significance (Risk ratio [RR], 0.75; CI, 0.56 to 1.01). Ha $e t$ als study is a meaningful study, but they did not stratify the patient's renal function, nor did they explore the impact of the severity of renal insufficiency on the efficacy and safety of OACs. Our results further confirm and reinforce Ha et al s findings. In the subgroup analyses of $\mathrm{CrCl}$ stratification, we did not find that there were significant differences in the efficacy and safety of DOACs among the three groups $(\mathrm{CrCl}$ of 30-50, 50-80 and $>80$ $\mathrm{mL} / \mathrm{min}$, respectively). However, it is still impossible to deny that the severity of renal insufficiency can affect the actions of OACs due to inadequate sample size and a lower event rate. The pairwise meta-analysis by Alhousani $e t a l^{31}$ included 10 RCTs and suggested that DOACs, VKA and low-molecular-weight heparin (LMWH) showed no significant difference in preventing recurrent VTEs among patients with CKD, but DOACs had a significantly lower risk of bleeding events irrespective of the level of renal impairment compared with VKAs. The conclusions were essentially consistent with the results of acute VTE treatment in our pairwise meta-analysis. Our analysis divided subjects into acute and extended treatment groups, which is consistent with the original study design and may be more suitable for clinical practice.

Prior to our network meta-analysis, some studies also observed the differences between DOACs. A network meta-analysis published in 2014 showed that rivaroxaban and apixaban had the lowest risk of bleeding compared with other therapeutic regimens, including LMWH with dabigatran and LMWH with edoxaban, in the treatment of acute VTE. ${ }^{32}$ Another Bayesian network meta-analysis published in 2015 showed that in the treatment of acute VTE, apixaban was superior to dabigatran, rivaroxaban and edoxaban in the reduction of major bleeding or clinically relevant non-major bleeding. ${ }^{33}$ More recently, a retrospective population-based cohort study involving 15254 patients with acute VTE showed that the use of apixaban was associated with a decreased risk of major bleeding compared with rivaroxaban. ${ }^{34}$ The study by Wang et $a l^{35}$ consisted of both direct and indirect analyses and only included four RCTs with 6003 patients in the analysis of patients with VTE. The results showed that rivaroxaban was safer than warfarin in patients with VTE with $\mathrm{CrCl}$ 30-79 mL/min, while apixaban's superiority regarding bleeding events was only presented in patients with VTE with $\mathrm{CrCl} 50-79 \mathrm{~mL} / \mathrm{min}$. All the data, including our finding, suggest that differences between DOACs are objective and that apixaban may have advantages in reducing the risk of bleeding compared with other DOACs. In our network meta-analysis, 10 RCTs with 37298 patients were included and the results appear to be more credible due to the increased study number and sample size.

When DOACs are used in patients with renal insufficiency, due to the difference in pharmacokinetic properties, especially the difference in renal clearance ratio, 3637 the difference in safety between them may become more obvious. In this case, DOACs with high renal clearance are more likely to accumulate in the body and cause bleeding than those with low renal clearance. However, our research results are contrary to this; that is, differences in DOACs were observed in the subgroup with normal renal function, but not in the subgroup with mild and moderate renal impairment. One possible explanation is that this contradiction is related to the huge differences in sample size and number of events among the three subgroups (the subgroup with $\mathrm{CrCl} 30-50 \mathrm{~mL} / \mathrm{min}$ : 2127 patients with 234 bleeding events; $50-80 \mathrm{~mL} / \mathrm{min}$ : 13496 patients with 1219 bleeding events; more than $80 \mathrm{~mL} / \mathrm{min}$ : 33041 patients with 2682 bleeding events), which led to a decline in statistical power in the first two subgroups, so that the differences between DOACs could not be detected sensitively. Therefore, it is impossible to conclude from this result that there is no difference in DOACs between these two subgroups with impaired renal function. In the future, it will be necessary to expand the sample size and conduct head-to-head RCTs between DOACs for further testing.

Pharmacokinetic studies suggested that the peak-totrough ratio of rivaroxaban $10-20 \mathrm{mg}$ once daily was approximately $10.4-13.8,{ }^{38}$ and the ratio of edoxaban (at a dose of $90 \mathrm{mg}$ once daily) was $25.8,{ }^{39}$ whereas the average ratios were 3 for apixaban $5 \mathrm{mg}$ two times per day ${ }^{40}$ and 1.88 for dabigatran $150 \mathrm{mg}$ two times per day. ${ }^{41}$ Peak-totrough ratios were similarly lower for the two times per day than the once daily dosing regimens, providing less 
fluctuation in drug exposure over the dosing interval. A separate analysis comparing DOACs dosed two times per day (dabigatran and apixaban) with those dosed once daily (rivaroxaban and edoxaban) in the atrial fibrillation population found a more favourable safety profile with DOACs dosed two times per day and speculated that the decreased peak-to-trough ratios afforded by two times per day DOACs probably played an important role. ${ }^{42}$ Our results of the network meta-analysis seem to confirm the results of this analysis.

Thus, the current study supports the use of DOACs for preventing recurrent VTE or VTE-related death with fewer bleeding events than warfarin in patients with acute VTE and $\mathrm{CrCl}$ greater than $30 \mathrm{~mL} / \mathrm{min}$ in clinical practice. For extended treatment of VTE in patients with different kidney functions, DOACs significantly reduced the risk of recurrent VTE or VTE-related death, while they should be prescribed with caution because of the increased bleeding risk compared with placebo/aspirin. Our study does not permit a definitive conclusion about the preferred DOAC based on low-quality evidence, although we found a trend of apixaban being associated with a reduced risk of bleeding events for patients with VTE with a $\mathrm{CrCl}$ of more than $80 \mathrm{~mL} / \mathrm{min}$. Detailed characterisation of individual patient risk profiles, careful selection of patients for OAC therapy and intensive monitoring and treatment of patients with atrial fibrillation and CKD may improve outcomes in this high-risk population.

To further verify our results, head-to-head comparative studies with high quality between different DOACs are needed. Currently, a study named 'the Comparisons of Bleeding Risk Between Rivaroxaban and Apixaban' for the treatment of acute VTE is underway (ClinicalTrials.gov identifier: NCT03266783) and has been widely noticed. ${ }^{43}$ Moreover, patients with severe renal insufficiency are at a particularly high risk of both thromboembolism and bleeding, but no high-quality evidence-based recommendations exist to guide the management of these patients. Further research in this area is needed.

In summary, the results of meta-analyses suggest that DOACs as a whole are similar to warfarin in reducing recurrent VTE and VTE-related death but are significantly superior to warfarin in reducing the risk of bleeding in the treatment of acute VTE. Furthermore, the possible effects of renal insufficiency on the efficacy and safety of DOACs have not been confirmed, which needs further study after expanding the sample size in the future. The results of network meta-analyses suggest that DOACs are heterogeneous in terms of safety, and the preferred agents of different DOACs remain inconclusive although our study showed that apixaban may be superior to other DOACs in reducing the risk of bleeding. In clinical practice, the use of OACs, including DOACs, to treat VTE in patients with renal insufficiency still needs to be very carefully and closely monitored.

Acknowledgements The authors would like to thank the authors of the studies they used to conduct this study.
Contributors Research idea and study design $-X S$ and YC. Data acquisition-XS and BY. Data analysis/interpretation-XS, LW and YC. Statistical analysis-XS. Manuscript drafting -XS. Revising manuscript-YC. Supervision or mentorshipYC and HC. Each author contributed important intellectual content during manuscript drafting or revision and accepts accountability for the overall work by ensuring that questions pertaining to the accuracy or integrity of any portion of the work are appropriately investigated and resolved. YC and XS take responsibility that this study has been reported honestly, accurately, and transparently; that no important aspects of the study have been omitted; and that any discrepancies from the study as planned have been explained. YC is responsible for the overall content as guarantor. The guarantor accepts full responsibility for the finished work and/ or the conduct of the study, had access to the data, and controlled the decision to publish.

Funding This work was supported by grants from the National Science Foundation of China (82000655) and Capital Foundation of Medical Developments (CFMD 2018-2-1051).

Competing interests None declared.

Patient consent for publication Not required.

Ethics approval This study does not involve human participants.

Provenance and peer review Not commissioned; externally peer reviewed.

Data availability statement All data relevant to the study are included in the article or uploaded as supplemental information.

Supplemental material This content has been supplied by the author(s). It has not been vetted by BMJ Publishing Group Limited (BMJ) and may not have been peer-reviewed. Any opinions or recommendations discussed are solely those of the author(s) and are not endorsed by BMJ. BMJ disclaims all liability and responsibility arising from any reliance placed on the content. Where the content includes any translated material, BMJ does not warrant the accuracy and reliability of the translations (including but not limited to local regulations, clinical guidelines, terminology, drug names and drug dosages), and is not responsible for any error and/or omissions arising from translation and adaptation or otherwise.

Open access This is an open access article distributed in accordance with the Creative Commons Attribution Non Commercial (CC BY-NC 4.0) license, which permits others to distribute, remix, adapt, build upon this work non-commercially, and license their derivative works on different terms, provided the original work is properly cited, appropriate credit is given, any changes made indicated, and the use is non-commercial. See: http://creativecommons.org/licenses/by-nc/4.0/.

ORCID iD

Yipu Chen http://orcid.org/0000-0002-5079-4334

\section{REFERENCES}

1 LV J-C, Zhang L-X. Prevalence and disease burden of chronic kidney disease. Adv Exp Med Biol 2019;1165:3-15.

2 Tritschler T, Kraaijpoel N, Le Gal G, et al. Venous thromboembolism: advances in diagnosis and treatment. JAMA 2018;320:1583-94.

3 Kayali F, Najjar R, Aswad F, et al. Venous thromboembolism in patients hospitalized with nephrotic syndrome. Am J Med 2008;121:226-30.

4 Tveit DP, Hypolite IO, Hshieh P, et al. Chronic dialysis patients have high risk for pulmonary embolism. Am J Kidney Dis 2002;39:1011-7.

5 Abbott KC, Cruess DF, Agodoa LYC, et al. Early renal insufficiency and late venous thromboembolism after renal transplantation in the United States. Am J Kidney Dis 2004;43:120-30.

6 Poli D, Zanazzi M, Antonucci E, et al. Renal transplant recipients are at high risk for both symptomatic and asymptomatic deep vein thrombosis. J Thromb Haemost 2006;4:988-92.

7 Cheung KL, Zakai NA, Folsom AR, et al. Measures of kidney disease and the risk of venous thromboembolism in the REGARDS (reasons for geographic and racial differences in stroke) study. Am J Kidney Dis 2017;70:182-90.

8 Agnelli G, Buller HR, Cohen A, et al. Oral apixaban for the treatment of acute venous thromboembolism. N Engl J Med 2013;369:799-808.

9 EINSTEIN-PE Investigators, Büller HR, Prins MH, et al. Oral rivaroxaban for the treatment of symptomatic pulmonary embolism. N Engl J Med 2012;366:1287-97.

10 Hokusai-VTE Investigators, Büller HR, Décousus $\mathrm{H}$, et al. Edoxaban versus warfarin for the treatment of symptomatic venous thromboembolism. N Engl J Med 2013;369:1406-15. 
11 Schulman S, Kearon C, Kakkar AK, et al. Dabigatran versus warfarin in the treatment of acute venous thromboembolism. $N$ Engl $\mathrm{J}$ Med 2009;361:2342-52.

12 Konstantinides SV, Torbicki A, Agnelli G, et al. 2014 ESC guidelines on the diagnosis and management of acute pulmonary embolism. Eur Heart J 2014;35:997-1053.

13 Kearon C, Akl EA, Ornelas J, et al. Antithrombotic therapy for VTe disease: chest guideline and expert panel report. Chest 2016;149:315-52.

14 Su X, Chen Y, Yan B. Comparative effectiveness and safety of different anticoagulant agents in patients with thromboembolic risk and chronic kidney disease: a systematic review and Bayesian network meta-analysis. prospero 2018 CRD42018090896. Available: http://www.crd.york.ac.uk/PROSPERO/display_record.php?ID= CRD42018090896

15 Moher D, Liberati A, Tetzlaff J, et al. Preferred reporting items for systematic reviews and meta-analyses: the PRISMA statement. PLoS Med 2009;6:e1000097.

16 Sterne JAC, Savović J, Page MJ, et al. Rob 2: a revised tool for assessing risk of bias in randomised trials. BMJ 2019;366:I4898

17 DerSimonian R, Laird N. Meta-Analysis in clinical trials. Control Clin Trials 1986;7:177-88.

18 Knapp G, Hartung J. Improved tests for a random effects metaregression with a single covariate. Stat Med 2003;22:2693-710.

19 Lu G, Ades AE. Combination of direct and indirect evidence in mixed treatment comparisons. Stat Med 2004;23:3105-24.

20 Brooks SP, Gelman A. General methods for monitoring convergence of iterative simulations. J Comput Graph Stat 1998:434-55.

21 Leucht S, Cipriani A, Spineli L, et al. Comparative efficacy and tolerability of 15 antipsychotic drugs in schizophrenia: a multipletreatments meta-analysis. Lancet 2013;382:951-62.

22 Guyatt GH, Oxman AD, Kunz R, et al. What is "quality of evidence" and why is it important to clinicians? BMJ 2008;336:995-8.

23 University of Bern. Confidence in Network Meta-analysis [computer program. Bern, Switzerland, 2017. cinema.ispm.ch

24 Nikolakopoulou A, Higgins JPT, Papakonstantinou T, et al. Cinema: an approach for assessing confidence in the results of a network meta-analysis. PLoS Med 2020;17:e1003082.

25 Agnelli G, Buller HR, Cohen A, et al. Apixaban for extended treatment of venous thromboembolism. N Engl J Med 2013;368:699-708.

26 EINSTEIN Investigators, Bauersachs R, Berkowitz SD, et al. Oral rivaroxaban for symptomatic venous thromboembolism. $N$ Engl J Med 2010;363:2499-510.

27 Schulman S, Kearon C, Kakkar AK, et al. Extended use of dabigatran, warfarin, or placebo in venous thromboembolism. $N$ Eng $J$ Med 2013;368:709-18

28 Weitz Jl, Lensing AWA, Prins $\mathrm{MH}$, et al. Rivaroxaban or aspirin for extended treatment of venous thromboembolism. N Engl J Med 2017;376:1211-22.

29 Goldhaber SZ, Schulman S, Eriksson H, et al. Dabigatran versus warfarin for acute venous thromboembolism in elderly or impaired renal function patients: pooled analysis of RE-COVER and RECOVER II. Thromb Haemost 2017;117:2045-52.
$30 \mathrm{Ha}$ JT, Neuen BL, Cheng LP, et al. Benefits and harms of oral anticoagulant therapy in chronic kidney disease: a systematic review and meta-analysis. Ann Intern Med 2019;171:181-9.

31 Alhousani M, Malik SU, Abu-Hashyeh A, et al. Using ora anticoagulants among chronic kidney disease patients to prevent recurrent venous thromboembolism: a systematic review and metaanalysis. Thromb Res 2021;198:103-14.

32 Castellucci LA, Cameron C, Le Gal G, et al. Clinical and safety outcomes associated with treatment of acute venous thromboembolism: a systematic review and meta-analysis. JAMA 2014;312:1122-35.

33 Cohen AT, Hamilton M, Mitchell SA, et al. Comparison of the novel oral anticoagulants apixaban, dabigatran, edoxaban, and rivaroxaban in the initial and long-term treatment and prevention of venous thromboembolism: systematic review and network meta-analysis. PLoS One 2015;10:e0144856.

34 Dawwas GK, Brown J, Dietrich E, et al. Effectiveness and safety of apixaban versus rivaroxaban for prevention of recurrent venous thromboembolism and adverse bleeding events in patients with venous thromboembolism: a retrospective population-based cohort analysis. Lancet Haematol 2019;6:e20-8.

35 Wang Z, Xiang Q, Hu K, et al. Comparison of the safety and efficacy of direct oral anticoagulants and warfarin in atrial fibrillation or venous thromboembolism in patients with renal impairment: systematic review, meta-analysis and network meta-analysis. Am J Cardiovasc Drugs 2021;21:643-57.

36 Potpara TS, Ferro CJ, Lip GYH. Use of oral anticoagulants in patients with atrial fibrillation and renal dysfunction. Nat Rev Nephrol 2018;14:337-51.

37 Weber J, Olyaei A, Shatzel J. The efficacy and safety of direct oral anticoagulants in patients with chronic renal insufficiency: a review of the literature. Eur J Haematol 2019;102:312-8.

38 Mueck W, Stampfuss J, Kubitza D, et al. Clinical pharmacokinetic and pharmacodynamic profile of rivaroxaban. Clin Pharmacokinet 2014;53:1-16.

39 Ogata K, Mendell-Harary J, Tachibana M, et al. Clinical safety, tolerability, pharmacokinetics, and pharmacodynamics of the novel factor Xa inhibitor edoxaban in healthy volunteers. J Clin Pharmacol 2010;50:743-53.

40 Frost C, Nepal S, Wang J, et al. Safety, pharmacokinetics and pharmacodynamics of multiple oral doses of apixaban, a factor Xa inhibitor, in healthy subjects. Br J Clin Pharmacol 2013;76:776-86.

41 Clemens A, Haertter S, Friedman J, et al. Twice daily dosing of dabigatran for stroke prevention in atrial fibrillation: a pharmacokinetic justification. Curr Med Res Opin 2012;28:195-201

42 Clemens A, Noack H, Brueckmann M, et al. Twice- or once-daily dosing of novel oral anticoagulants for stroke prevention: a fixedeffects meta-analysis with predefined heterogeneity quality criteria. PLoS One 2014;9:e99276.

43 ClinicalTrials.gov. Us national library of medicine. Available: https:// www.clinicaltrials.gov/ct2/show/NCT03266783 